\title{
A New Approach towards Clustering based Color Image Segmentation
}

\author{
Dibya Jyoti Bora \\ Department of Computer Science \& Applications \\ Barkatullah University,Bhopal-462026(M.P.)
}

\begin{abstract}
:
Color image segmentation is currently a very emerging topic for researchers in Image processing. Clustering is a frequently chosen methodology for this image segmentation task. But for a better segmentation, there arises the need of an optimal technique. In this paper, we propose an integrated approach for color image segmentation which is a new of its kind. Here, we integrate the famous k-means algorithm with watershed algorithm. But, here we chose 'cosine' distance measure for k-means algorithm to optimize the segmented result of the later one. Also, as color space has a leading impact on color image segmentation task, so, we chose HSV color space for our proposed approach. Since usually the noise arises during the segmentation process, so here the final segmented image is filtered by median filter to make the output image clearer and noise free. The result of the proposed approach is found to be quite satisfactory.
\end{abstract}

\section{General Terms:}

Image Processing, Color Image Segmentation

\section{Keywords:}

Image Segmentation, Color Image Segmentation, HSV

Color space, K Means, Cosine Distance, Watershed

Algorithm.

\section{INTRODUCTION:}

Image segmentation is the major part of image processing research. This can be defined as the process of partitioning a digital image into multiple segments which may carry more meaningful information and hence make it easy to analyze[1][2]. These segments can be considered homogenous according to some criterion such as color, motion, texture etc [3]. Images may be either color or gray scale images. So, depending on the type of the image, image segmentation may be either color image segmentation or gray scale image segmentation [4]. Color image segmentation is found to be very useful in many applications. There exist so many techniques for image segmentation tasks. But, we have adopted clustering methodology. Clustering is an unsupervised study which is done to separate the data into different groups known as clusters [5]. But on doing so, two properties are maintained: 1. High Intra cluster property and 2. Low Inter Cluster Property. According to the first property, objects inside the same cluster should exhibit high similarity. And the second property says that objects of one cluster should be different from the objects reside in another cluster. Clustering is found to be very popular methodology for the image segmentation task [4]. We have chosen K-Means algorithm

\author{
Anil Kumar Gupta, Ph.D. \\ Department of Computer Science \& Applications \\ Barkatullah University,Bhopal-462026(M.P.)
}

among the available ones because of its simplicity [5]. Also we focus on the distance measure used in the clustering algorithm [6]. "Cosine" distance measure is found to be very well suitable for the color image segmentation task [3]. As we are concerned with color image segmentation, so, we are giving importance on choosing a proper color space. As HSV color space is found to be more similar to the way the human eyes perceive color, so, we preferred this color space for our color image segmentation task [7]. First RGB image is converted into HSV one. Then K Means algorithm is applied on the resultant image. The segmented image is filtered with sobel filter. The resulting image is then analyzed with marker controlled watershed method to have the final segmented image. The final segmented image is filtered by median filter to remove the noises occurred during the whole segmentation process thereby obtaining our required colored segmented image of the original image. The paper is organized as follows: First of all we have presented a review on the previous work done in the area. Then, the flowchart of the proposed methodology is shown. After that a clear illustration of all the topics involved in the proposed approach are described. Then, we come to the experiment section and finally, the conclusion section.

\section{REVIEW OF LITERATURE:}

In [7], the authors proposed a color image segmentation approach where they first converted the rgb image into hsv one. Then they applied Otsu's multi-thresholding on Vchannel to get the best threshold from the image. The resultant image is then segmented with K-Means clustering to merge the over segmented regions that occurred due to the application of Otsu's multi-thresholding. Finally they performed background subtraction along with morphological processing. The result of the approach is found to be quite satisfactory as per the values of MSE and PSNR obained from the experiment.

In [8], the authors first converted the original image from RGB form to HSV form. Then they applied mean shift and FELICM separately on Hue, Saturation and Value Components. The final images obtained from mean shift and FELICM is fused together. The proposed method show better performance level than the previous algorithms.

In [9], the authors proposed a new quantization technique for HSV color space to generate a color histogram and a gray histogram for K-Means clustering, which operates across different dimensions in HSV color space. In this approach, the initialization of centroids and the number of cluster are automatically estimated. A filter for postprocessing is introduced to effectively eliminate small spatial regions. This method is found to achieve high 
computational speed and the results are closed to human perceptions. Also with this method, it becomes possible to extract salient regions of images effectively.

In [10], the authors presented a modified watershed method where they used Convolution filtering, Dynamic thresholding and Masking, Morphology operations on input image. The proposed method is compared with Region Growing and FCM algorithms and found a better performance of the former one.

In [11], the authors analyzed the properties of the hsv color space with emphasis on the visual perception of the variation in Hue, Saturation and Intensity values of an image pixel. They extracted pixel features by either choosing the Hue or the Intensity as the dominant property based on the Saturation value of a pixel. The segmentation using this method gives better identification of objects in an image compared to those generated using RGB color space.

In [12], authors performed a comparative study between integrated k-means algorithm and watershed algorithm with integrated fuzzy c means algorithm and watershed algorithm and found the performance of the former one better with respect to the segmentation result.

\section{FLOWCHART OF THE PROPOSED APPROACH:}

The proposed approach can be represented in the flow chart as follows:

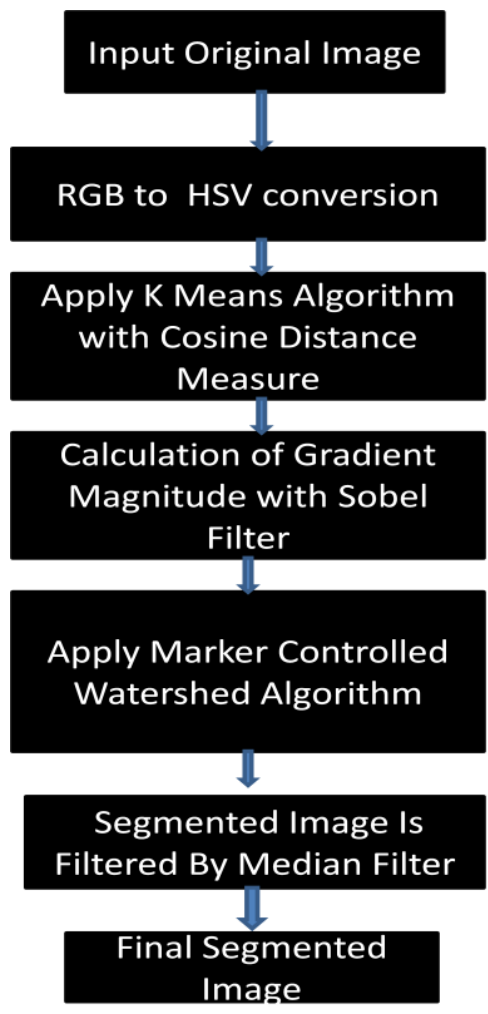

Fig 1: Flow Chart of the Proposed Approach

\section{HSV COLOR SPACE:}

A HSV color space can be represented using a hexacone in three dimensions where the central vertical axis represents the intensity [12]. Here ' $\mathrm{H}$ ' stands for 'Hue'. The "Hue" is defined as an angle in the range $[0,2 \pi]$ relative to the red axis with red at angle 0 , green at $2 \pi / 3$, blue at $4 \pi / 3$ and red again at $2 \pi[13][14]$. ' $S$ ' stands for 'Saturation'. The Saturation describes how pure the hue is with respect to a white reference. It can be thought of as the depth or purity of color and is measured as a radial distance from the central axis with values between 0 at the center to 1 at the outer surface. For $\mathrm{S}=0$, as one moves higher along the intensity axis, one goes from black to white through various shades of gray. On the other hand, for a given intensity and hue, if the saturation is changed from 0 to 1 , the perceived color changes from a shade of gray to the most pure form of the color represented by its hue[14]. ' $\mathrm{V}$ ' stands for 'Value'. Value is a percentage that goes from 0 to 100 . This range (from 0 to 100) can be thought as the amount of light illuminating a color [15]. For example, when the hue is red and the value is high, the color looks bright. On the other hand, when the value is low, it looks dark. A Diagrammatic view of the HSV color space is [16]:

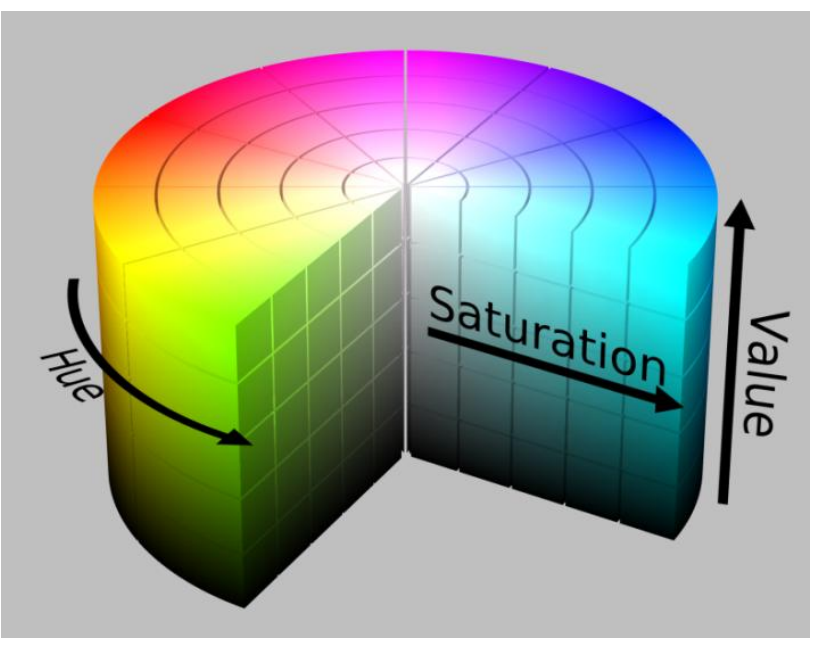

Fig 2: HSV Color Space

\section{K- MEANS ALGORITHM:}

This is a famous hard clustering algorithm best known for its simplicity [5].Usually hard clustering algorithm involves much less mathematical computation in comparison to soft clustering algorithm. In case of soft clustering algorithms, it is also a critical issue to determine the fuzziness factor [17]. These are the reasons, why we have gone for hard clustering algorithm and chosen K-Means algorithm. The main steps involved in K-Means algorithm are [18]:

[a] At first, we select $\mathrm{k}$ initial clusters(randomly chosen)

[b] Then we assign all the objects to their closest clusters.

[c] Re compute the centroid of each cluster

[d] Repeat [b] and [c] until the centroids do not change or memberships finalize. 
In this algorithm, we try to minimize the following objective function (known as squared error function)[18][19]:

$J=\sum_{j=1}^{k} \sum_{i=1}^{n}\left\|x_{i}^{(j)}-c_{j}\right\|^{2}$

where $\left\|x_{i}^{(j)}-c_{j}\right\|^{2}$ is a chosen distance measure between a data point $x_{i}^{(j)}$ and the cluster centre $c_{j}$, is an indicator of the distance of the $n$ data points from their respective cluster centers.

A diagrammatic view of the algorithm is[20] :

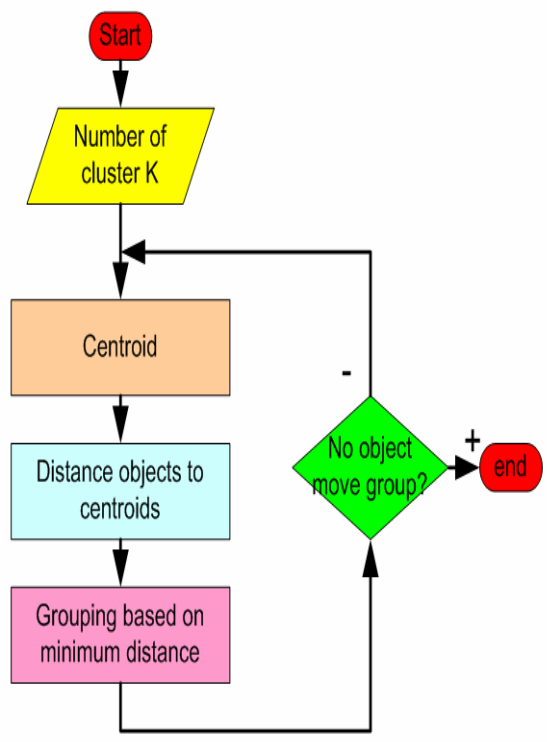

Fig 3: K Means Algorithm

\section{COSINE DISTANCE MEASURE:}

This distance measure is a measure of similarity between two vectors of $n$ dimensions by finding the cosine of the angle between them. Cosine distance measure is based on the measurement of operation and not magnitude [21]. As this distance measure devotes much towards the orientation of data points which is much more related to our work for dealing with pixels of an image, so we have chosen this to be used in K-Means algorithm. Mathematically, it can be defined as : Given an $m$-by- $n$ data matrix $\mathrm{X}$, which is treated as $m(1-$ by- $n)$ row vectors $\mathrm{x} 1, \mathrm{x} 2, \ldots, \mathrm{x} m$, the cosine distances between the vector $\mathrm{x} s$ and $\mathrm{x} t$ are defined as follows[22] :

$$
d_{s t}=1-\frac{x_{s} x_{t}^{\prime}}{\sqrt{\left(x_{s} x_{s}^{\prime}\right)\left(x_{t} x_{t}^{\prime}\right)}}
$$

\section{SOBEL FILTER:}

In image processing or computer vision, sobel filter comes under edge detection algorithms [24]. It is actually a discrete differentiation operator which computes an approximation of the gradient of the image intensity function. When we apply sobel operator to an image, then the result will be either the corresponding gradient vector or norm of the vector at each point of the image. With each image the sobel operator involves two kernels $G_{x}$ and $G_{y}$. Here, $G_{x}$ is estimating the gradient in $x$-direction while $G_{y}$ estimating the gradient in $y$-direction.

Then the absolute gradient magnitude will be given by:

$|\mathbf{G}|=\sqrt{ }\left(\mathbf{G}_{\mathbf{x}}{ }^{2}+\mathbf{G}_{\mathbf{y}}{ }^{2}\right)$

Although, this value is often approximated with [24]:

$|\mathbf{G}|=\left|\mathbf{G}_{\mathbf{x}}\right|+\left|\mathbf{G}_{\mathbf{y}}\right|$

Sobel operator has a powerful smoothing effect on the random noises of an image. It is differentially separated by two rows and columns, so the edge elements on both sides become enhanced resulting a very bright and thick look of the edges[24]. These are the reasons why we have selected sobel operator for our work.

\section{MARKER-CONTROLLED WATERSHED SEGMENTATION:}

The watershed algorithm is first proposed by Digabel and Lantuejoul[25,26,27]. This algorithm considers a grey level image as a topographic relief. If we combine the grey level of each point at an altitude then it is possible to define the watershed transform as the ridge forming the boundary between two watersheds [28]. In this way, watershed of the said relief can be computed. This corresponds to regions of the image. But the problem associated with watershed algorithm is the over segmentation problem [25][26]. The over segmentation problem means creations of too many regions. This over segmentation problem can be solved with the following two methods [28]:

(1) Hierarchical watershed segmentation [29].

(2) Watershed by markers [30]

In hierarchical approach, a tree of regions is generated from the result of the watershed [28]. First, the regions and watershed are merged. Next, the hierarchical segmentation process merges the regions with lowest borders. At last we will have a tree which provides us the possibility to explore the different levels of fusion regions [29].

In our approach we have adopted Watershed by markers.

The Marker-Controlled Watershed Segmentation consists of the following steps [31]

1. First, a segmentation function is computed which is an image whose dark regions are the objects we are trying to segment.

2. Foreground markers are computed. These are connected blobs of pixels within each of the objects. 3 . Then compute background markers. These are pixels that are not part of any object. 4. Modify the segmentation function so that it only has minima at the foreground and background marker locations.

5. And at last the watershed transform of the modified segmentation function is calculated. 


\section{MEDIAN FILTER:}

This is a non linear method used to remove noise from images [32][33]. Salt and pepper noise can be easily reduced by median filter. One of the most powerful features of this filter is its effectiveness at removing noise while preserving edges. This feature is the reason why median filter is frequently chosen in image segmentation task as we know the rule played by edges in the same. The working methodology of this filter is: it moves through the image pixel by pixel replacing each value by median value of the neighboring pixels [33]. The pattern of the neighbors is called the "window", which slides, pixel by pixel, over the entire image. The median is calculated by first sorting all the pixel values from the window into numerical order. Then replace the considering pixel with the median pixel value.

\section{EXPERIMENTS \& RESULTS:}

The proposed approached has been implemented in Matlab. The images for the experiment are collected from the Matlab demo images with image processing tool box. We have taken three images: onion, football and pears [34]. Number of clusters is set to three. The results obtained during the segmentation process are shown below clearly in a sequence for each of the images:

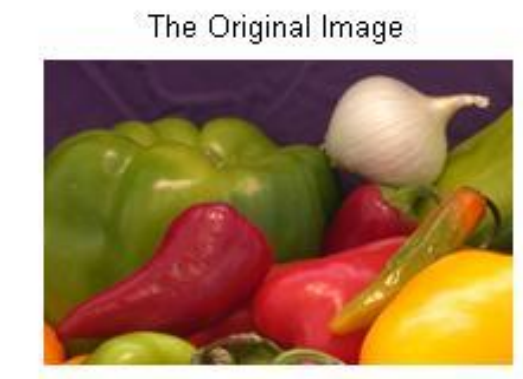

Fig 4.1 : Original Onion Image

The Image in HSV Color Space

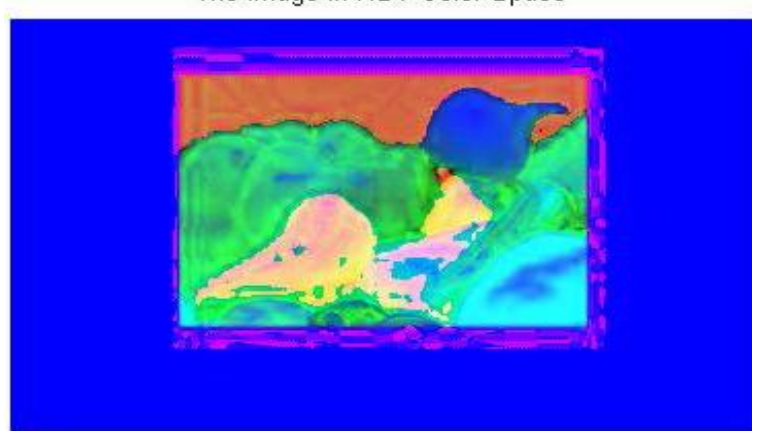

Fig 4.2 : The Image in HSV Color Space

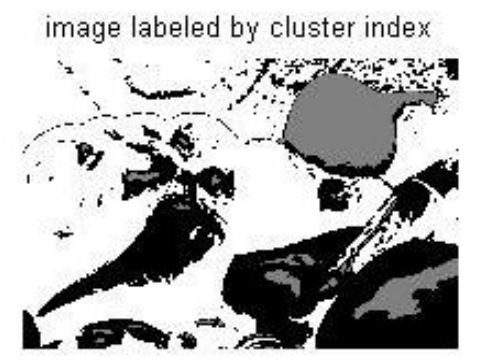

Fig 4.3: Image after K Means Clustering

Gradient magnitude (gradmag)

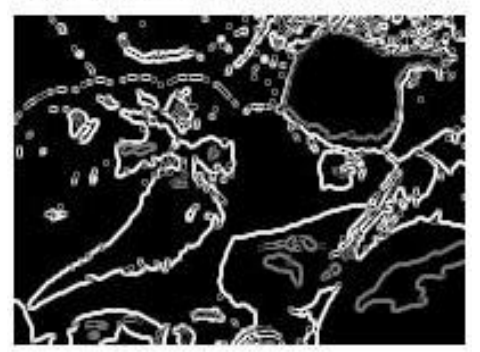

Fig 4.4: Image obtained after Applying Sobel operator

Watershed transform of gradient magnitude (Lrgb)

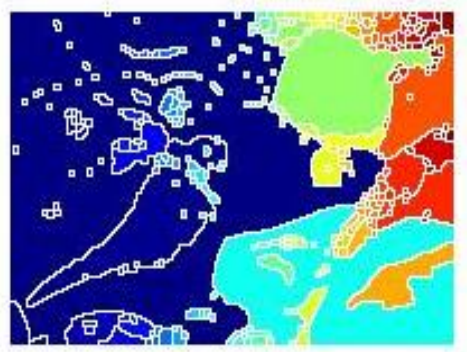

Fig 4.5: Image Obtained After Applying Watershed Algorithm

Image Filtered By Median Filter

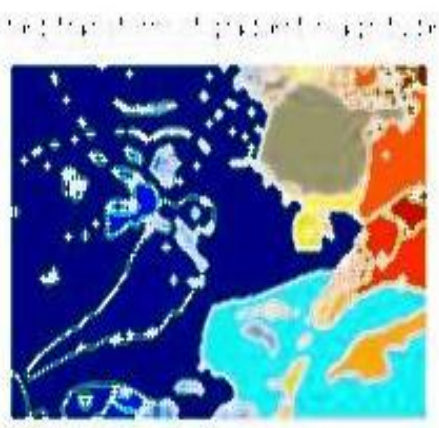

Fig 4.6: Final Segmented Image obtained After Applying Median Filter 


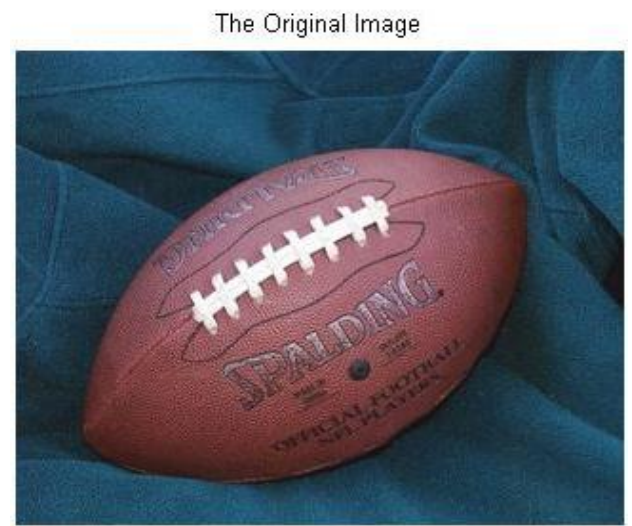

Fig 5.1: Original Football Image

The Image in HSV Color Space

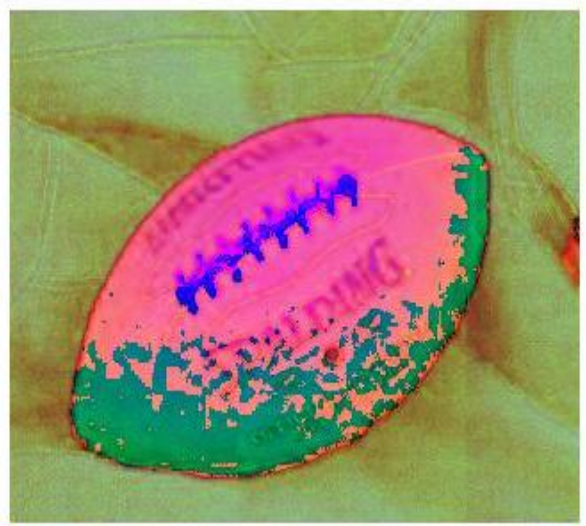

Fig 5.2: The Image in HSV Color Space Algorithm image labeled by cluster index

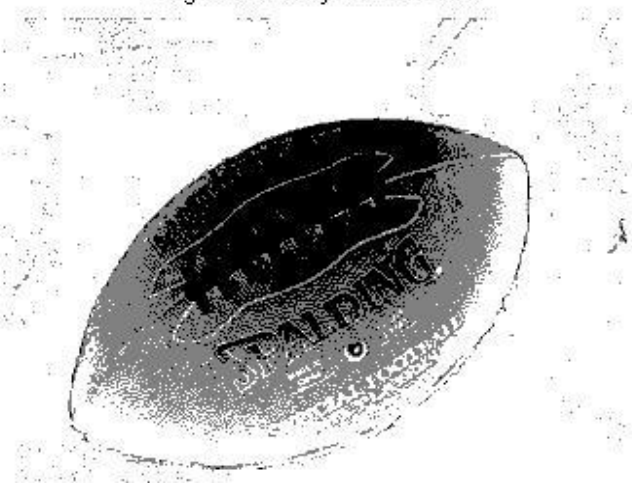

Fig 5.3: Image after K Means Clustering

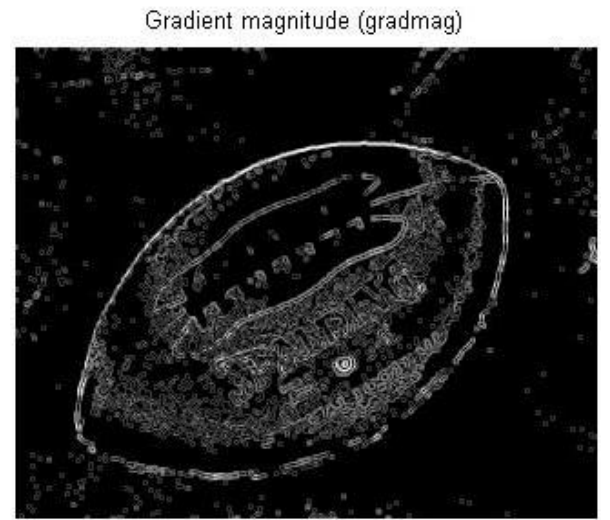

Fig 5.4: Image obtained after Applying Sobel operator

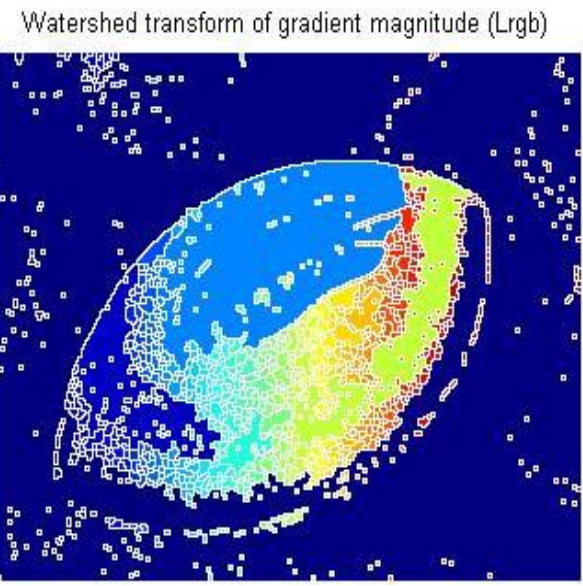

Fig 5.5: Image Obtained After Applying Watershed Algorithm

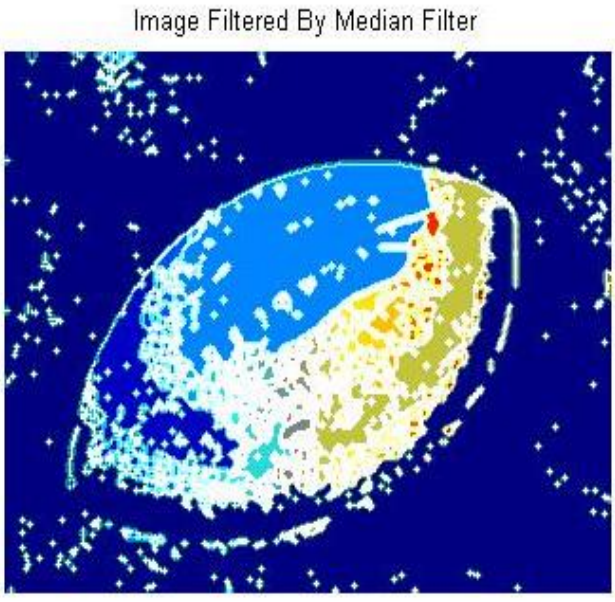

Fig 5.6: Final Segmented Image obtained After Applying Median FiltMedian Filter 


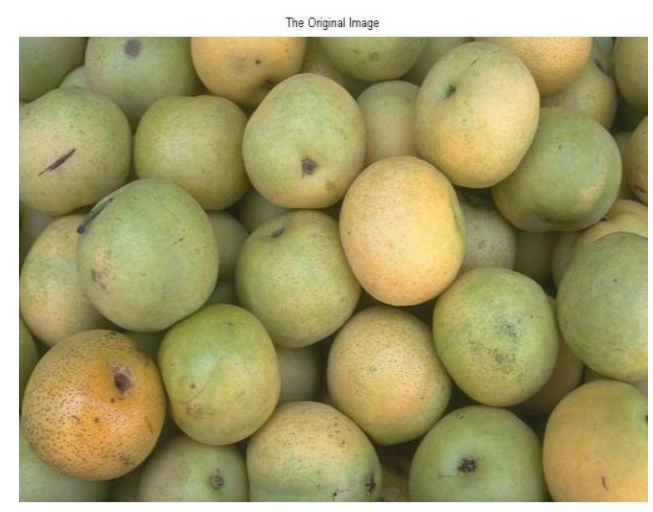

Fig 6.1: Original Pears Image

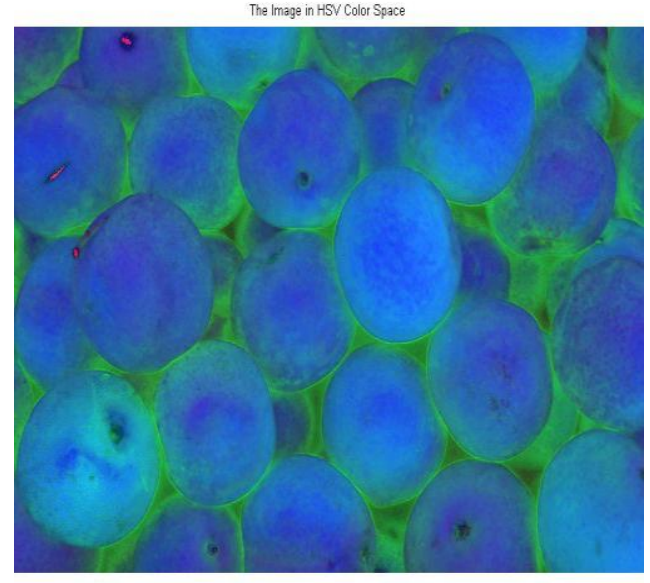

Fig 6.2: The Image in HSV Color Space

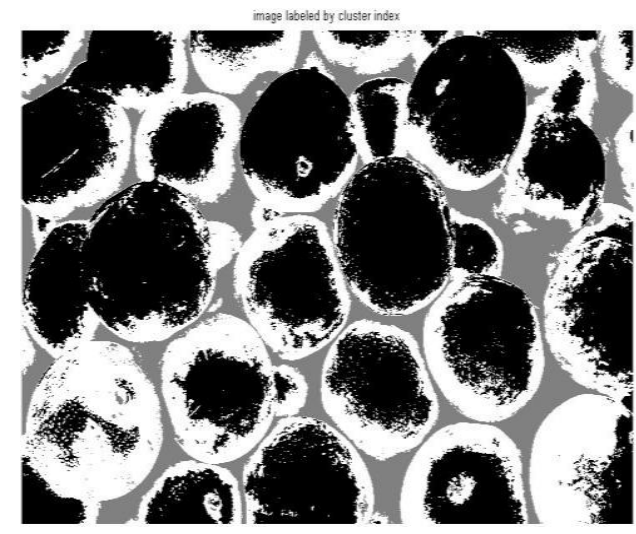

Fig 6.3: Image after K Means Clustering

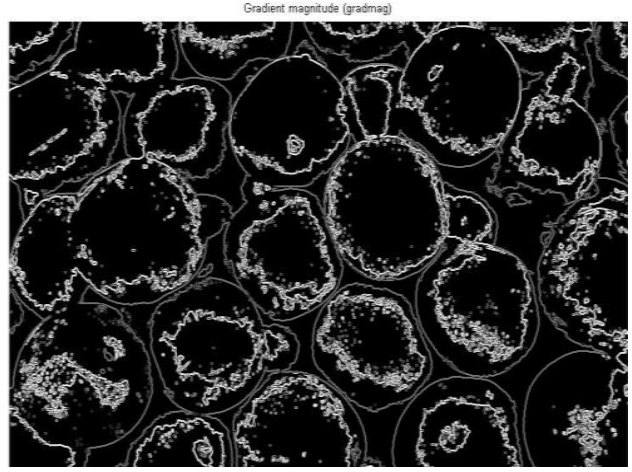

Fig 6.4: Image obtained after Applying Sobel operator

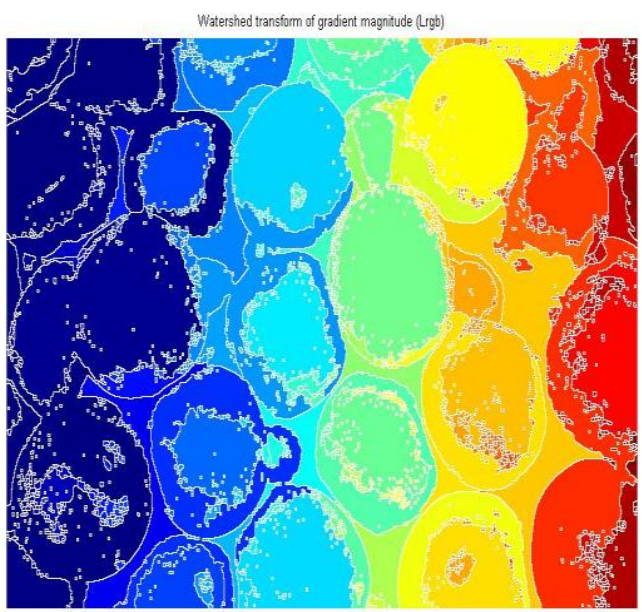

Fig 6.5: Image Obtained After Applying Watershed Algorithm

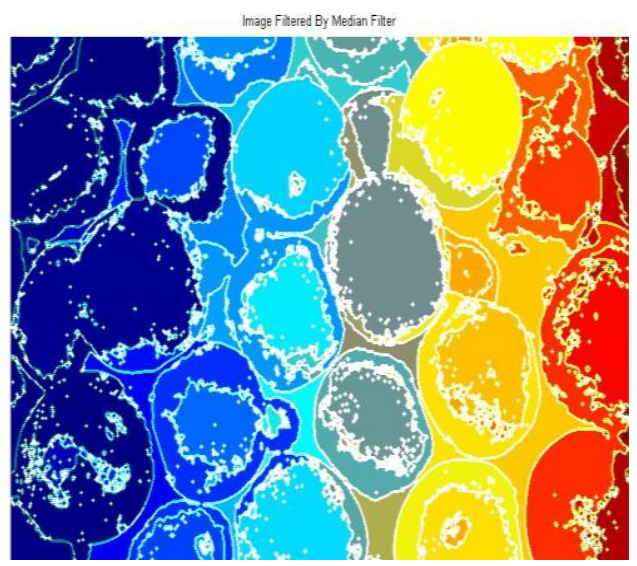

Fig 6.6: Final Segmented Image obtained After Applying Median Filter

So, from the resultant final segmented images of each of the original images, it is clearly visible that our proposed approach succeeds to produce a satisfactory result for color based image segmentation. 


\section{CONCLUSION:}

Color image segmentation has the capability to enhance the image analysis process which always improves the segmentation result. But, here choosing a proper color space is a very important issue. So, we have selected HSV for this purpose. Our proposed approach is a kind of novel approach in the color image segmentation field. In this approach, we have brought the new concept of integrating two clustering algorithm with proper post processing of the segmented image. Post processing is in the sense that we filtered the final segmented image with median filter. This is utmost needed as noises may arise during the whole segmentation process. Our segmentation results found to be clear with more distinct visibility of the edges. So, in future we go for applying this approach in medical imaging area. Also our future research will focus on developing a technique for determining the number of clusters automatically so that an incorrect assumption of the same will not hamper the segmentation process.

\section{REFERENCES:}

[1] Linda G. Shapiro and George C. Stockman (2001): "Computer Vision", New Jersey, Prentice-Hall, ISBN 0-13-030796-3, pp 279-325

[2] Barghout, Lauren, and Lawrence W. Lee. "Perceptual information processing system.", Paravue Inc. U.S. Patent Application 10/618,543, filed July 11, 2003

[3] Dibya Jyoti Bora, Anil Kumar Gupta, "A Novel Approach Towards Clustering Based Image Segmentation", International Journal of Emerging Science and Engineering (IJESE), ISSN: 2319-6378, Volume-2 Issue-11, September 2014, pp. 6-10.

[4] Dibya Jyoti Bora, Anil Kumar Gupta, "Clustering Approach Towards Image Segmentation: An Analytical Study", International Journal Of Research In Computer Applications And Robotics,ISSN 23207345 Vol.2 Issue.7, July 2014, pp. 115-124 .

[5] Dibya Jyoti Bora, Anil Kumar Gupta, "A Comparative study Between Fuzzy Clustering Algorithm and Hard Clustering Algorithm", International Journal of Computer Trends and Technology (IJCTT) - volume 10 number 2 -Apr 2014, pp. 108-113.

[6] Dibya Jyoti Bora, Anil Kumar Gupta, "Effect of Different Distance Measures on The Performance of K-Means Algorithm: An Experimental Study in Matlab", International Journal of Computer Science and Information Technologies (IJCSIT), Vol. 5 (2), 2014, pp.2501-2506

[7] Vijay Jumb, Mandar Sohani, Avinash Shrivas, "Color Image Segmentation Using K-Means Clustering and Otsu's Adaptive Thresholding", International Journal of Innovative Technology and Exploring Engineering (IJITEE) ISSN: 2278-3075, Volume-3, Issue-9, February 2014, pp. $72-76$

[8] Amanjot Kaur Randhawa, Rajiv Mahajan, "An Improved Approach towards Image Segmentation Using Mean Shift and FELICM", International Journal of Advanced Research in Computer Science and Software Engineering, Volume 4, Issue 7, July 2014, pp. 197-202

[9] Tse-Wei Chen; Yi-Ling Chen; Shao-Yi Chien, "Fast image segmentation based on K-Means clustering with histograms in HSV color space," Multimedia Signal Processing, 2008 IEEE 10th Workshop on, vol., no. 810 Oct. 2008, pp.322-325.

[10] Rupinder Kaur, Garima Malik, "Modified Watershed Transform using Convolution filtering for Image Segmentation", International Journal Of Engineering Sciences \& Research Technology, volume 3(8): August, 2014, pp. 449-454.

[11] S. Sural, G. Qian, and S. Pramanik, "Segmentation and histogram generation using the HSV color space for image retrieval," , Proceedings of IEEE International Conference on Image Processing, Sep. 2002, pp. 589592.

[12] M.C. Jobin Christ ,R.M.S. Parvathi, "Segmentation of Medical Image using Clustering and Watershed Algorithms", American Journal of Applied Sciences 8 (12),2011,pp. 1349-1352

[13] G.Stockman and L.Shapiro, "Computer Vision", Prentice Hall, 2001

[14] S. Sural, G. Qian, and S. Pramanik, "Segmentation and histogram generation using the HSV color space for image retrieval," presented at IEEE International Conference on Image Processing, Rochester, New York, 2002

[15] http://www.dig.cs.gc.cuny.edu/manuals/Gimp2/Grokki ng-the-GIMP-v1.0/node51.html

[16] http://commons.wikimedia.org/wiki/File\%3AHSV_col or_solid_cylinder_alpha_lowgamma.png

[17] Dibya Jyoti Bora, Anil Kumar Gupta, "Impact of Exponent Parameter Value for the Partition Matrix on the Performance of Fuzzy C Means Algorithm", International Journal of Scientific Research in Computer Science Applications and Management Studies, Volume 3, Issue 3 (May 2014).

[18] A.K. Jain, M. N. Murty, P. J. Flynn, "Data Clustering: A Review",ACM Computing Surveys, vol. 31, Sep. 1999, pp. 264-323.

[19] A.K. Jain, "Data clustering: 50 years beyond Kmeans", Pattern Recognition Letters, Elsevier, 2010

[20] http://people.revoledu.com/kardi/tutorial/kMean

[21] Singhal, Amit,"Modern Information Retrieval: A Brief Overview",Bulletin of the IEEE Computer Society Technical Committee on Data Engineering 24 (4), 2001, pp. 35-43.

[22] http://www.mathworks.in/help/stats/pdist.html

[23] Irwin Sobel, 2014, History and Definition of the Sobel Operator

[24] Raman Maini ,Dr. Himanshu Aggarwal , "Study and Comparison of Various Image Edge Detection 
Techniques “, International Journal ofImage Processing (IJIP), Jan-Feb 2009, Volume (3) Issue (1), pp.1-11.

[25] Serge Beucher, "Watershed, hierarchical segmentation and waterfall algorithm", Mathematical morphology and its applications to image processing, Kluwer Academic Publishers, 1994,pp 69-76.

[26] S. Beucher, "Unbiased Implementation of the Watershed Transformation based on Hierarchical Queues”,CMM Internal note, Paris 206 School of Mines, 2004

[27] H. Digabel et C. Lantuejoul. Iterative algorithms. Dans 2nd European Symp. Quantitative Analysis of Microstructures in Material Science, ,Biology and Medicine. Caen, France, 1978, pp. 85-99.

[28] Aliiaout, A. and M. Nasri, 2012. Medical image segmentation by marker controlled watershed and mathematical morphology. Int. J. Multimedia Applic., 4, DOI: 10.5121/ijma.2012.4301, pp. 1-9.

[29] P. Arbeláez, M. Maire, C. Fowlkes and J. Malik."Contour Detection and Hierarchical Image
Segmentation", IEEE TPAMI, Vol. 33, No. 5, May 2011,,pp. 898-916.

[30] K. Parvati, “ Image Segmentation Using Gray-Scale Morphology and Marker-Controlled watershed Transformation", Discrete Dynamics in Nature and Society, vol. 2008, Article ID 384346, 8 pages, 2008.

[31] Mohmed Ali Hamdi, "Modified Algorithm Marker Controlled Watershed Transform For Image Segmentation Based On Curvelet Threshold",Canadian Journal On Image Processing and Computer Vision, Vol. 2 No. 8, Dec. 2011

[32] Zeinab A. Mustafa, Banazier A. Abrahim and Yasser M. Kadah, "Modified Hybrid Median Filter for Image Denoising", 29th National Radio Science Conference, April-2012.

[33] Usha Mittal1, Sanyam Anand, "Modified Watershed Segmentation with Denoising of Medical Images", International Journal of Innovative Research in Science, Engineering and Technology, Vol. 2, Issue 4, April 2013,pp. 982-987.

[34] http://www.mathworks.in/products/image 